\title{
Measurement of Adhesion Forces between Particles and Rough Substrates in Air with the Vibration Method ${ }^{\dagger}$
}

\author{
Siegfried Ripperger ${ }^{1}$, Konrad Hein ${ }^{2}$ \\ ${ }^{1}$ Lehrstuhl für Mechanische Verfahrenstechnik, \\ Technische Universität Kaiserslautern* \\ ${ }^{2}$ Lehrstuhl für Mechanische Verfahrenstechnik, \\ Technische Universität Dresden**
}

\begin{abstract}
Pull-off forces were measured using the vibration method at 30-50\% relative humidity for glass and tin spheres on a variety of substrates. The results were compared with those obtained through the colloidal probe technique. Both methods show good agreement for small particle sizes. Since the vibration method causes sinusoidally alternating stresses, the method yields detachment and contact forces between particles and substrate of the same order of magnitude. Alternating contact forces of the vibration method can cause an increase in the adhesion force through flattening of asperities, which also depend on the surface roughness and the mechanical properties of particle and substrate.

Pull-off force measurements with the colloidal probe technique and special attention to the influence of the contact force also show an adhesion force intensification with increasing contact forces depending on the surface roughness. No significant adhesion force intensification caused by increasing contact time to 30s at several contact forces was observed. For theoretical predictions based on van der Waals adhesion, an approach presented by Rabinovich and approximations of plastic microasperity flattening were combined.
\end{abstract}

\section{Introduction}

The accuracy of dosing procedures with fine powders in the pharmaceutical industry or the removal of abrasive particles after polishing operations are examples for industrial operations which are significantly influenced by adhesion phenomena in particle-wall systems. Such adhesion phenomena can arise from several adhesion mechanisms such as van der Waals adhesion, capillary or electrostatic adhesion forces, whose intensity depends on the topography, the chemical composition of the interacting surfaces, environmental conditions such as relative humidity and temperature, and the stresses between the surfaces in contact in combination with their mechanical properties.

Different techniques have been developed to measure the adhesion forces between particles and substrate. The so-called "colloidal probe technique" [1,

\footnotetext{
* 67653 Kaiserslautern, Germany

**01062 Dresden, Germany

${ }^{\dagger}$ Accepted: August 26, 2004
}

2], based on the atomic force microscope (AFM) [3], and the centrifuge technique $[4,5]$ have become wellestablished tools for studying adhesion forces. The vibration method $[6,7]$ with its technical conversion described later represents a complementary and practical method for the measurement of adhesion forces. Its alternating detachment and contact forces have a practical counterpart in the dynamic stresses acting on particles during frequently used operations of process engineering.

The paper presents adhesion force measurements between particles and rough substrates obtained through the vibration method at room temperature and moderate relative humidity between $30 \%$ and $50 \%$. Under these ambient conditions, van der Waals adhesion represents the dominating adhesion mechanism. The results of the vibration method are compared with those obtained by the colloidal probe technique. We focus on the influence of surface roughness and contact forces on adhesion forces, while taking deformations of micro-asperities through alternating contact forces of the vibration method into account. 
Single particles were placed on the substrate using gentle sieve vibration to disperse the particles from the powder, avoiding any appreciable triboelectrical loading. The actuator with the mounted substrate was placed at the end of the laminar inlet zone of a grounded aluminium flow channel with rectangular cross-section over a drilling at the bottom side of the channel. With increasing acceleration of the substrate, the acting detachment force exceeds the adhesion force. Particle detachment events are continuously surface were measured spectrally with a laser-scanning vibrometer (PSV 200, Polytec, Germany).

\subsection{The vibration method}

The measuring principle of the vibration method, first described by Deryaguin [6], is based on particle detachment from a vertical-sinusoidally vibrating surface caused by its inertia at a certain acceleration. Therefore, the vibration of the substrate not only yields a detachment force to overcome the adhesion force, but also causes contact forces between particles and substrate of the same order. Particle detachment events are continuously recorded and correlated with acting acceleration and particle mass, allowing the pull-off forces to be calculated.

\subsubsection{Technical conversion}

Our technical conversion differs in several aspects from that of Deryaguin. The use of a laser-scanning vibrometer (LSV) for the spectral measurement of the mode shape and acceleration of the vibrating substrate can be seen as the main difference. Figure 1 shows the experimental set-up for the measurement of adhesion forces between particles and substrate. In contrast to our earlier investigations [7], the sinusoidal oscillation of the substrate is realized by a preloaded open-loop ultrasonic piezo actuator (UPA 25, US 100, LC 100; Cedrat Technologies, France) with adjustable frequency and excitation voltage as well as a temperature sensor. The test substrate $(5 \times 5 \mathrm{~mm})$ is mounted above an adapter which is attached to the actuator over a prestressed screwed fastening and a small amount of epoxy resin, thus preventing additional vibration modes between the three components. The actuator provides a maximum acceleration of approximately $100000 \mathrm{~g}$ at its resonance frequency, which also depends on the actuator temperature and the mass of the mounted substrate. For that reason, the acceleration calibration of the sinusoidally oscillating substrate was carried out as a function of the excitation voltage $\mathrm{U}_{\text {ex }}$ and actuator temperature at fixed frequency $f$ as shown in Figure 2.

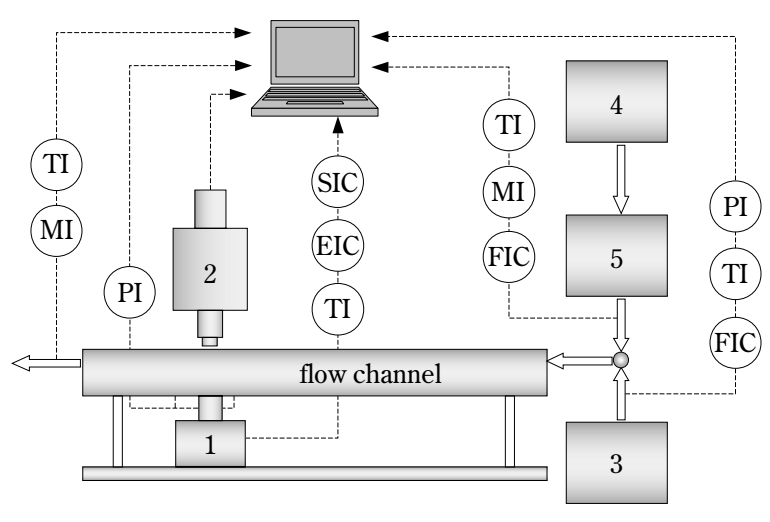

Fig. 1 Experimental set-up: (1) oscillation system with attached substrate; (2) optical unit with microscope, CCD camera and image analysing software; (3) compressed air with $\mathrm{T}_{\text {dew }}=-60^{\circ} \mathrm{C}$; (4) laminar flow box; (5) climatic exposure test cabinet and flow channel $(\mathrm{L} \times \mathrm{W} \times \mathrm{H}: 1700 \times 40 \times 4 \mathrm{~mm})$.

excitation voltage actuator $\mathrm{U}_{\mathrm{ex}}[\mathrm{V}]$

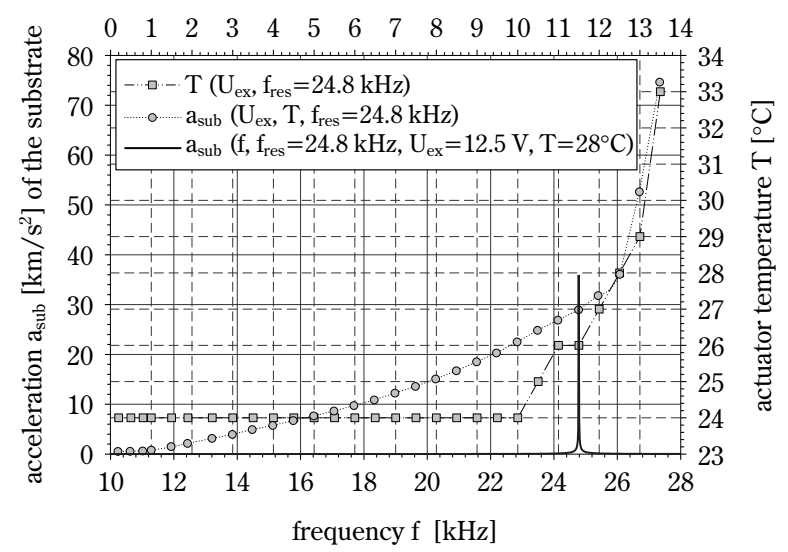

Fig. 2 Acceleration calibration curve $\mathrm{a}_{\mathrm{sub}}\left(\mathrm{U}_{\mathrm{ex}}\right)$ of an electropolished stainless steel substrate. Additionally, the frequencydependent course of $\mathrm{a}_{\text {sub }}$ (f) and the heating of the actuator as a function of the excitation voltage $T\left(U_{e x}\right)$ at fixed frequency are plotted. 
rocorded through a glass plate attached over the substrate at the top side of the flow channel with a microscope (Axiotech, Zeiss, Germany), a CCD camera and an image analysis software (Image Pro Plus, Media Cybernetics, USA). For horizontal dislocation of detached particles, the substrate is exposed to a laminar air flow $\left(\operatorname{Re}_{\text {channel }}=1514\right)$ parallel to the substrate. The air flow was cleaned and conditioned with a laminar-flow box (VMK 06.15, Steag, Germany), a climatic exposure test cabinet (KPK 200, Feutron, Germany) and a frequency-controlled fan. Optionally, compressed air with a dew point temperature of $\mathrm{T}_{\mathrm{dew}}=-60^{\circ} \mathrm{C}$ can be used to realize very low relative humidity conditions. Flow rate, air temperature and humidity were measured continuously with a mass flowmeter (3063, TSI, USA), and a humidity sensor (FH A646, Ahlborn, Germany). Velocity profiles over the channel crosssection at different air flow rates were quantified with a hot-wire probe (8465, TSI, USA).

The adhesion force between particle and substrate can also be measured using particle re-entrainment in a turbulent air flow. In this case, a turbulent flow regime parallel to the nonvibrating surface is used to apply drag and lift forces to the adhering particles.

\subsubsection{Principle of force measurement}

Figure 3 shows a schematic diagram of the forces acting on a single particle exposed to a laminar air flow and adhered to a sinusoidally oscillating surface. Particle re-entrainment caused by fluid forces is determined by the stresses within the linear sublayer of the laminar flow $\left(\operatorname{Re}_{\text {channel }}=1514\right)$. The estimation of acting lift $\left(\mathrm{F}_{\text {lift }}\right)$ and drag $\left(\mathrm{F}_{\mathrm{drag}}\right)$ forces for a particle fully submerged in the linear sublayer in contact with

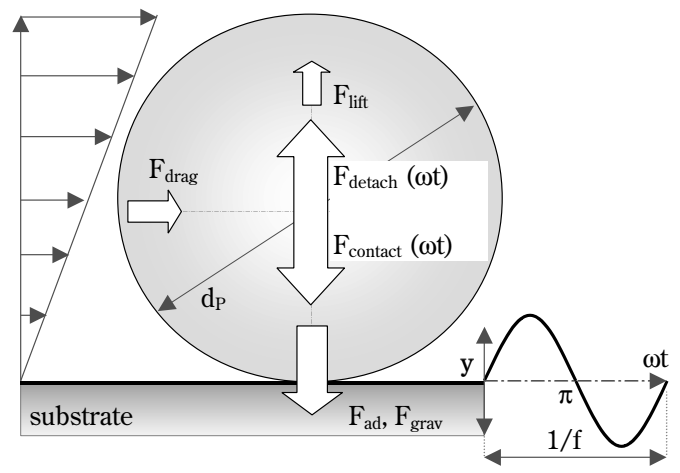

Fig. 3 Forces acting on a single particle exposed to a laminar air flow and adhered to a sinusoidally oscillating substrate (schematic). a wall based on approaches by Leighton [8] and O'Neill [9] is described in detail by Hein et al. [7]. Under the given laminar flow conditions and particle size range, the contribution of both lift and drag forces to particle detachment as well as the gravitational force are negligible in relation to the adhesion forces and detachment forces. This was confirmed by force measurements with particle re-entrainment in a turbulent air flow at the same particles and nonvibrating substrates under otherwise unchanged environmental conditions. Under laminar flow conditions, fluid forces are only used for the horizontal dislocation of detached particles.

The maximum detachment force $\mathrm{F}_{\text {detach }}$ and contact force $\mathrm{F}_{\text {contact }}$ are sinusoidally alternating inertial forces of the same order of magnitude acting on the particle at the top and bottom dead centre of the sinusoidally oscillating substrate.

$$
\left|\mathrm{F}_{\text {detach }}\right|=\left|\mathrm{F}_{\text {contact }}\right|
$$

Hence, the acceleration of the particle acts in the direction opposite to the acceleration of the vibrating surface, which is given by:

$$
\mathrm{a}_{\mathrm{sub}}(\mathrm{t})=-\mathrm{a}_{\mathrm{P}}(\mathrm{t})
$$

The absolute value of the maximum detachment and contact force results from the particle mass and the acceleration of the sinusoidally oscillating substrate at particle detachment, which is measured and calibrated with the LSV (Laser-scanning-vibrometer) as described in Section 2.1.1.

$$
\left|\mathrm{F}_{\text {detach }}\right|=\left|\mathrm{F}_{\text {contact }}\right|=\left|\mathrm{a}_{\text {sub }}\right| \cdot \mathrm{m}_{\mathrm{P}}
$$

The mass of a spherical particle is calculated from its projected area and density. A simplified force balance at the top dead centre of the sinusoidally oscillating substrate under the assumptions of negligible small fluid forces permits calculation of the pull-off force between particle and substrate as follows:

$$
\mathrm{F}_{\text {detach }}=\mathrm{F}_{\mathrm{ad}}
$$

The maximum contact force acts on the particle at the bottom dead centre. Its order of magnitude can be approximated under the assumption of an additive superposition of adhesion force and contact force [10] and substituting $\mathrm{F}_{\mathrm{ad}}$ and $\mathrm{F}_{\text {contact }}$ from Eq. (1) and (4).

$$
\mathrm{F}_{\text {contact_max }} \approx \mathrm{F}_{\text {ad }}+\mathrm{F}_{\text {contact }} \approx\left|\mathrm{F}_{\text {detach }}\right|+\mathrm{F}_{\text {contact }} \approx 2 \cdot\left|\mathrm{F}_{\text {detach }}\right|
$$

\subsection{Comparison with other methods}

As discussed in Section 1, the adhesion force and 
the underlying adhesion mechanisms are influenced by various factors. The different measurement principles of each technique and their technical conversion result in different stresses and contact times between the interacting surfaces. This leads to a wide range of reported values for adhesion between the same particle-substrate system depending on the technique employed. In the light of this aspect, we give a brief comparison of the colloidal probe technique, centrifuge method and vibration method.

The measuring principle of the vibration method causes alternating detachment and contact forces of the same order of magnitude at the top and bottom dead centre of the sinusoidally oscillating substrate. In contrast, the colloidal probe technique or the centrifuge method apply a uniform and incremental detachment force to the particles and a contact force can be applied independently, just before particle detachment.

The maximum rotational speed of the ultra-centrifuge and the maximum oscillation amplitude of the sinusoidally oscillating piezo actuator limits the maximum detachment force especially for the vibration method. Depending on the acting adhesion force, particle density and the optical acquisition of the single particles, measurements with both methods are restricted to a minimum particle size of a few micrometres. Since the particles cannot be monitored continuously during the increase of the centrifuge's rotational speed, the precise moment of particle detachment events or the precise adhesion force cannot be determined. In most cases, the centrifuge must be stopped after each run at constant centrifugal acceleration. Since the attachment of particles to the AFM cantilever is usually done using micromanipulators under an optical microscope, the minimal particle size is limited to approximately $1 \mu \mathrm{m}$ [11]. This comparatively tedious and high effort for sample preparation also limits the number of separate particles used within one study for practical reasons [11]. In contrast, the centrifuge technique and the vibration method usually measure the detachment force between various particles at different substrate locations in a single experiment, resulting in good statistical evaluation of the data. The colloidal probe technique allows the use of the same particle for several experiments at different substrate locations, and the particle surface topography can be subsequently examined. A further difference can be seen in the contact time between particle and substrate before particle detachment. With the centrifuge technique and the vibration method, the interacting surfaces usually remain in contact for more than 1 minute, whereas the colloidal probe technique normally uses a measuring frequency of $1 \mathrm{~Hz}$ for repeated force measurements.

\section{Test Particles and Substrates}

Adhesion force measurements were carried out for glass spheres (Potters-Ballotini, UK), and tin spheres (Nanoval, Germany) on ground and electropolished stainless steel substrates (Lehrstuhl für Maschinenund Apparatekunde, TU München, Germany) as well as a silicon wafer substrate (Institut für Halbleiterund Mikrosystemtechnik, TU Dresden, Germany). The topography and surface roughness of the test particles and the substrate were characterized by SEM (Figure 4, Figure 5) and AFM (Figure 6). Particles and substrates were cleaned with ethanol in an ultrasonic bath, rinsed in deionised water and dried in an exsiccator. The substrates were additionally cleaned carefully with a low-pressure stream of compressed air before each measurement. Further attempts to clean the surfaces were not made to match its practical use. Table 1 presents relevant material properties and results of the AFM roughness analysis.

\subsection{Roughness analysis of particles and substrates}

The root mean square roughness (rms) represents one possibility of quantifying the surface topography by means of an average value. This standardized roughness parameter was incorporated by Rabinovich [12] into the classic approach of Rumpf [13], to account for roughness effects on van der Waals adhesion (see Section 4). Since the rms values themselves do not indicate the frequency of the roughness or their characteristic peak-to-peak distance between the asperities, it is dependent on scale. Further approaches have been developed to account for this dependency and an asperity shape differing from the hemisphere [12]. Especially for real, technical surfaces with inhomogeneities caused by the manufacturing process, determination of the dominating roughness scale(s) and their associated roughness parameters out of a three-dimensional surface topography remains demanding. The box plot of the rms roughness against the scanning area allows information to be extracted about the dependency of the surface roughness on scale, taking variations of surface roughness caused by the manufacturing process into consideration. Figure $\mathbf{7}$ shows the relationship between rms roughness and the area of scanning for stainless steel substrates and particles measured by AFM at 

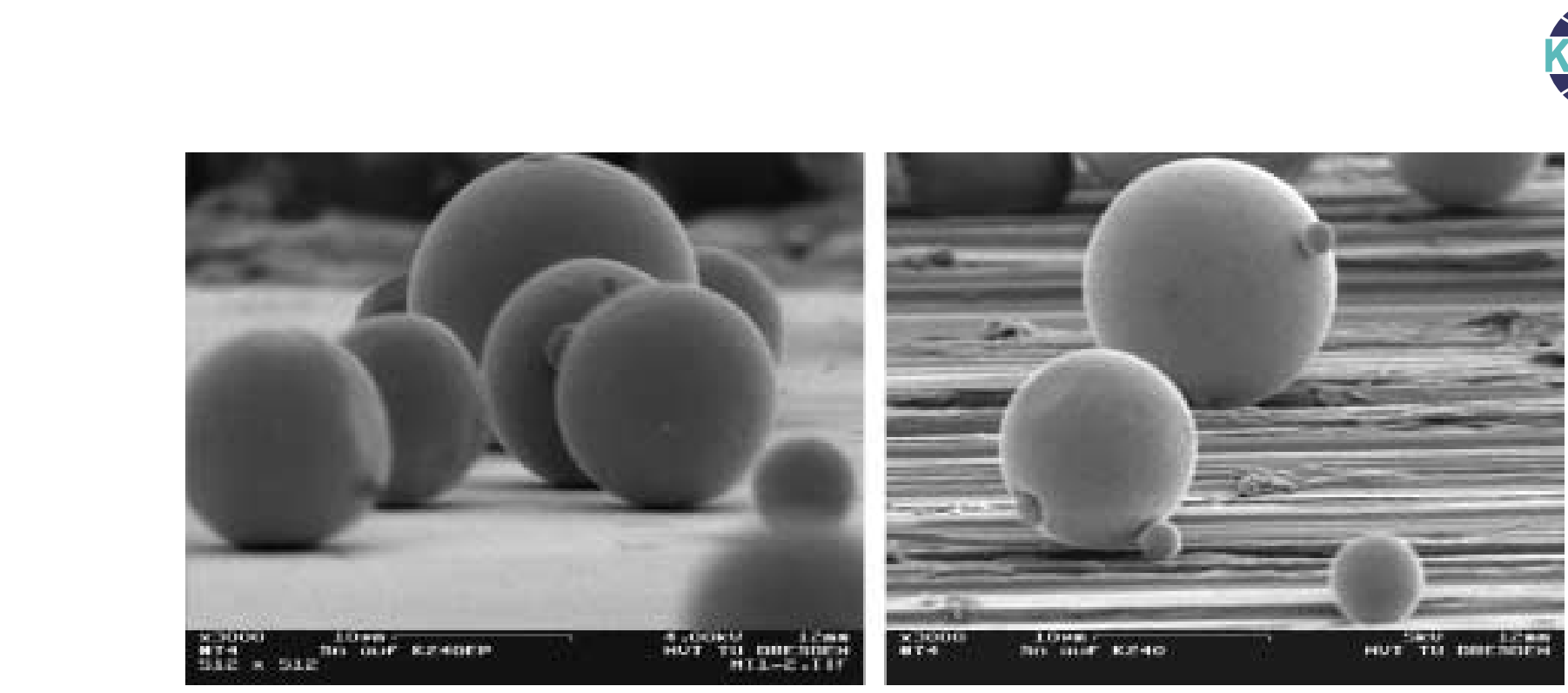

Fig. 4 SEM images of tin particles placed on an electropolished stainless steel substrate (left) and of tin particles placed on a ground stainless steel substrate (right).
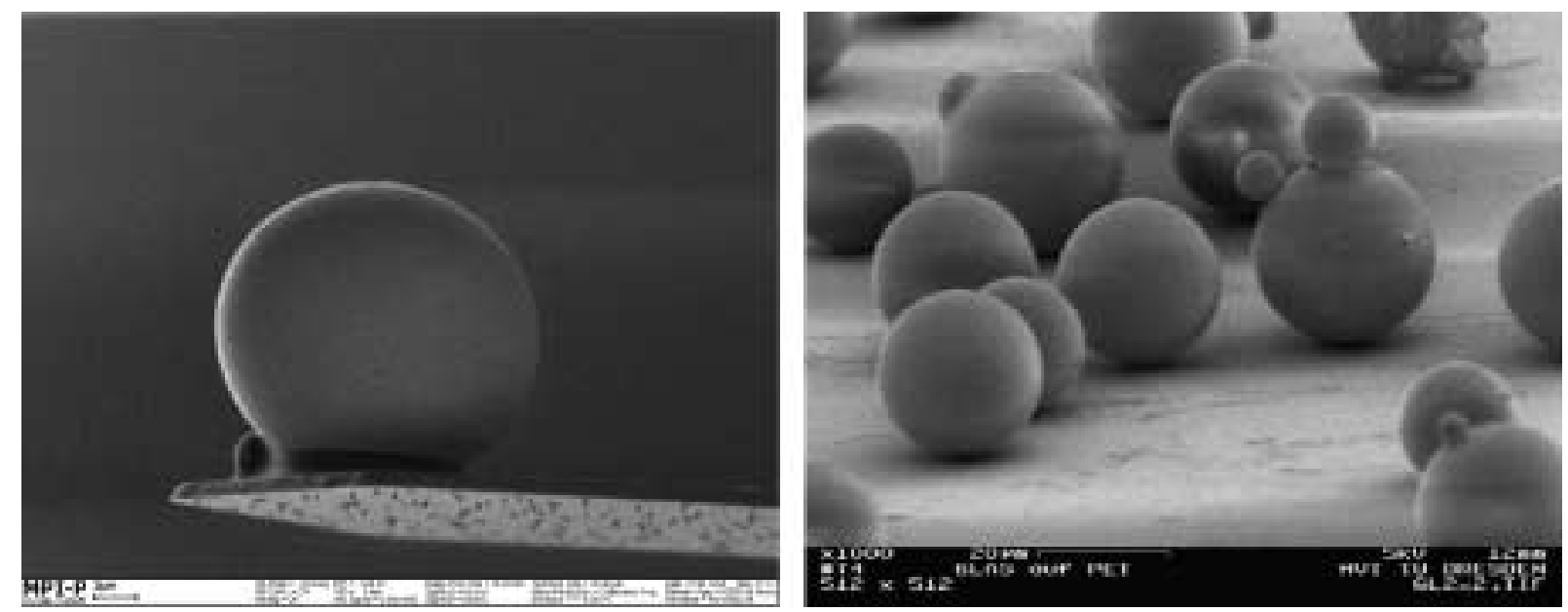

Fig. 5 SEM images of a tin particle attached to an AFM cantilever (left, MPI for Polymer Research, Mainz) and glass spheres on a PET substrate (right).

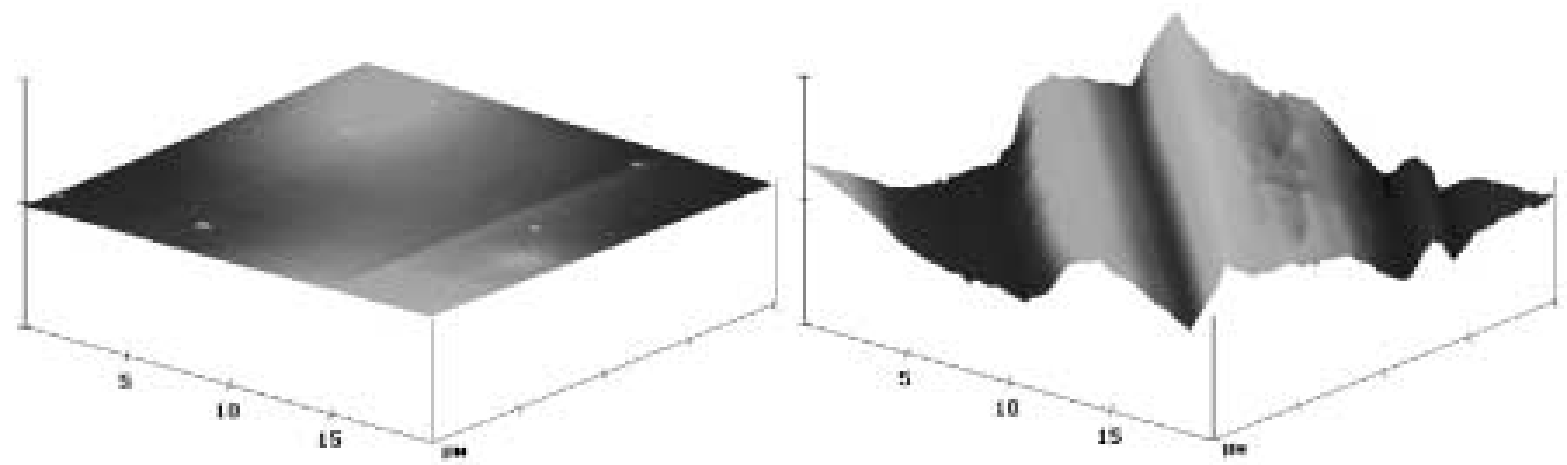

Fig. 6 AFM images of $20 \times 20 \mu \mathrm{m}$ area with a vertical scale of $3000 \mathrm{~nm} /$ division of electropolished (left) and ground stainless steel substrate (right). 


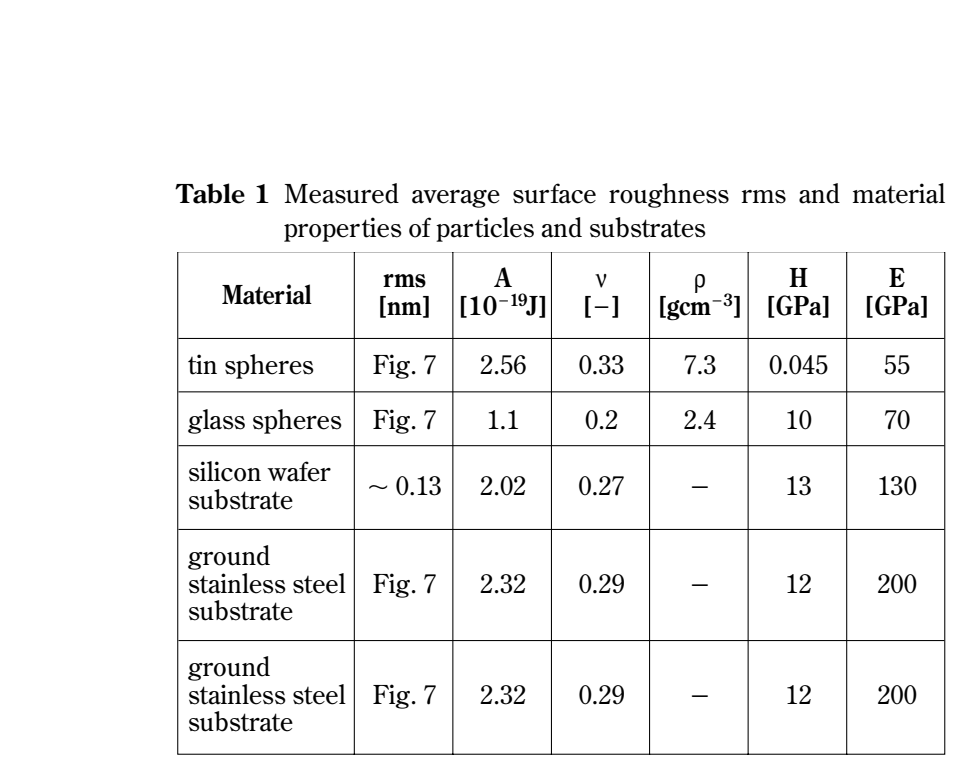

various positions. Depending on the asperity distribution and size, the scanned surfaces show an increase in rms roughness with increasing scanning area tending toward a characteristic maximum rms roughness. Similarly, a characteristic minimum scanning area exists, where only a nanoscopic roughness scale is dominant, as described previously by Kiely [14]. Despite the large variations in surface roughness for the ground stainless steel substrate, a significant reduction in rms roughness for very small scanning areas can be observed. This possibly indicates the existence of a "microscopic" roughness within the macroscopic dimension. Owing to its dependency on scale, use of the rms roughness in the approaches presented by Rabinovich raises the issue of a proper

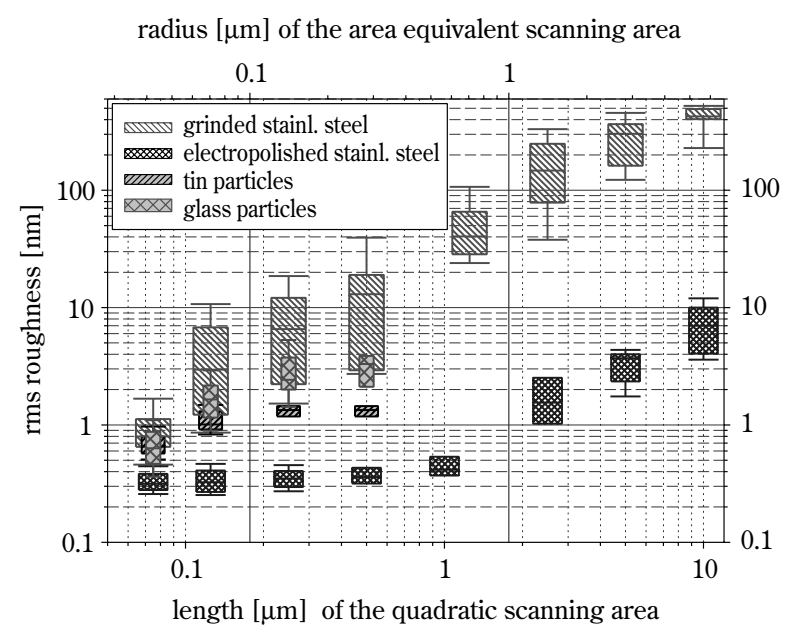

Fig. 7 rms-roughness analysis for ground and electropolished stainless steel substrates and tin and glass particles at different scanning areas and different positions calculated from AFM images. choice of the scanning area to reflect the topography of the interacting surfaces in the real area of contact during force measurements.

\section{Prediction of Adhesion Forces}

A number of approaches exist for the prediction of particle-substrate interactions for ideally smooth surfaces, based on van der Waals adhesion [15], or derived from surface-energy-based approaches such as Derjaguin-Muller-Toporov (DMT) [16], or JohnsonKendall-Roberts (JKR) [17] adhesion theory. The DMT and JKR theory take the elastic flattening effect into account. A major drawback towards the applicability of the DMT and JKR model lies in the need for an estimation of surface energy. Surface energy values vary depending on the source and its measurement technique, since influences of surface roughness or plastic deformation are often coupled within the surface energy parameter [18]. For a van-der-Waalsbased approach, the fundamental interaction parameters are much better established. Although not accounting for polar forces, it can be assumed that the major contribution to surface energy results from van der Waals attraction.

With real surfaces, the roughness influence on the real area of contact and the magnitude of the force of adhesion limits the application of these models. A possibility of overcoming this problem is by simulating the influence of roughness on the force of adhesion, based on the local topography and chemical composition of the interacting surfaces $[19,20]$. Other approaches try to quantify the surface topography by means of average values. Rabinovich [12] incorporated the rms roughness in the approach by Rumpf [13] to account for the effects of hemispherical asperities on van der Waals adhesion:

$$
\mathrm{F}_{\mathrm{vdW}}=\frac{\mathrm{A}_{12} \cdot \mathrm{d}_{\mathrm{P}}}{12 \cdot \mathrm{a}_{0}^{2}} \cdot\left(\frac{1}{1+\frac{\mathrm{d}_{\mathrm{P}}}{2.97 \cdot \mathrm{rms}_{12}}}+\frac{1}{\left(1+\frac{1.48 \cdot \mathrm{rms}}{\mathrm{a}_{0}}\right)^{2}}\right)
$$

The effective Hamaker constant $A_{12}$ between material 1 and 2 in vacuum can be calculated from the individual Hamaker constants of the adhesion partners as follows:

$$
\mathrm{A}_{12}=\sqrt{\mathrm{A}_{1} \cdot \mathrm{A}_{2}}
$$

The effective $\mathrm{rms}_{12}$ roughness of the interacting surfaces is calculated from the rms values of the substrate $\mathrm{rms}_{\text {sub }}$ and the particle $\mathrm{rms}_{\mathrm{P}}$ (Fig. 7), assuming 


$$
\mathrm{rms}_{12}=\sqrt{\mathrm{rms}_{\mathrm{P}}^{2}+\mathrm{rms}_{\mathrm{sub}}^{2}}
$$

This approach was enhanced repeatedly to account for an asperity shape differing from the hemisphere, superposition of roughness scales and finally elastic flattening of asperities [12], resulting in closer predictions of experimental adhesion values. On the other hand, these approaches are associated with more roughness parameters whose prediction might be complex and prone to subjective error, especially for anisotropic surfaces stemming from the manufacturing process. In the light of these findings, we use the approach of Eq. (6) in combination with a statistical and scale-dependent roughness characterization (section 3.1) of the particle and substrate to account for the topography of the interacting surfaces in the real area of contact. This should allow for the prediction of adhesion forces with a minimum of individual interpretation error during roughness characterization of the surfaces.

\subsection{Influence of contact forces}

Since the vibration method causes alternating detachment and contact forces of the same order of magnitude, the influence of contact forces on the adhesion forces should be regarded. According to Rumpf and co-workers [14], an adhesion force intensification caused by the elastic flattening of microasperities is only possible through an increase of the points of contact, however, a visco-elastic [21] or plastic flattening of the contact area can also intensify the adhesion force.

An estimation of plastic deformation can be based on various criteria. Kogut [22] and Maugis [23] suggested a comparison of a critical flattening or a critical contact radius with the corresponding elastic values. Depending on the degree by which the critical value is exceeded, elastic or elasto-plastic deformations take place. Rumpf [14] proposes a comparison of the contact pressure and yield strength Y or material hardness $\mathrm{H}$ to detect the elasto-plastic or plastic deformation of the contact area.

To consider the effect of micro-asperity plastic flattening on van der Waals adhesion we combine the Rabinovich approach (Eq. (6)) together with an estimation of plastic deformation by Rumpf. Because of the mechanical properties of the adhesion partners (Table 1), we discuss in all cases the adhesion between a soft, deformable particle and an approximately rigid substrate. Under this assumption, micro- asperity plastic flattening can be considered to be a plastic flattening of the particle $h_{\text {flat_plast, }}$ expressed through a modified effective roughness $\mathrm{rms}_{12}$ plast in Eq. (6):

$$
\mathrm{rms}_{12 \_ \text {plastic }}=\sqrt{\left(\mathrm{rms}_{\mathrm{P}}-\mathrm{h}_{\mathrm{flat} \text { _plast }}\right)^{2}+\mathrm{rms}_{\text {sub }}^{2}}
$$

Assuming only normal stresses and elastic contact at the circular interface between a smooth soft particle and a smooth rigid substrate, the contact pressure distribution $\mathrm{p}_{\text {contact }}(\mathrm{r})$ over the contact radius $r$, the maximum elastic contact radius $r_{\text {contact }}$ and the elastic flattening $\mathrm{h}_{\mathrm{flat}}$ for a given contact force $\mathrm{F}_{\text {contact }}$ can be estimated with the Hertz theory [24].

$$
\begin{aligned}
& \mathrm{p}_{\text {contact }}(\mathrm{r})=\frac{3 \cdot \mathrm{F}_{\text {contact }} \cdot \sqrt{1-\left(\frac{\mathrm{r}}{\mathrm{r}_{\text {contact }}}\right)^{2}}}{2 \cdot \pi \cdot \mathrm{r}_{\text {contact }}^{2}} \\
& \mathrm{r}_{\text {contact }}^{3}=\frac{\mathrm{F}_{\text {contact }} \cdot \mathrm{d}_{\mathrm{P}}}{2 \cdot \mathrm{K}_{\text {elast }}\left(\mathrm{E}_{\mathrm{P}}, \mathrm{E}_{\text {sub }}, \mathrm{v}_{\mathrm{P}}, \mathrm{v}_{\text {sub }}\right)}
\end{aligned}
$$

$$
\mathrm{h}_{\mathrm{flat}}=\frac{2 \cdot \mathrm{r}_{\mathrm{contact}}^{2}}{\mathrm{~d}_{\mathrm{P}}}
$$

According to Rumpf [14], the maximum contact force $F_{\text {contact_max }}$ consists of the adhesion force of the unstressed system and the external contact force $\mathrm{F}_{\text {contact }}$ In the case of the vibration method, the maximum contact force $\mathrm{F}_{\text {contact_max }}$ can be estimated with Eq. (5). For the condition

$$
\mathrm{p}_{\text {contact }}\left(\mathrm{r}, \mathrm{F}_{\text {contact_max }}\right) \geq \mathrm{H}_{\mathrm{P}}
$$

a plastically deformed contact radius $r_{\text {contact_plast }}$ $\left(\mathrm{p}_{\text {contact }}=\mathrm{H}_{\mathrm{P}}\right)$ and a plastic flattening $\mathrm{h}_{\mathrm{flat} \text { plast }}$ of the particle can be calculated, rewriting Eq. (10) and sub-

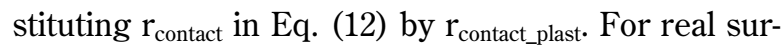
faces, roughness will decrease the real area of contact $\mathrm{A}_{\text {contact }}=2 \cdot \pi \cdot \mathrm{r}_{\text {contact }}^{2}$ and thus increase the contact pressure, resulting in a stronger plastic flattening. One attempt to account for this effect, considering the deformable particle only, uses the bearing function available in the standard software of the AFM. This function uses AFM topography images to calculate the ratio $a_{\text {real }}(h)$ of the real contact area $A_{\text {real }}$ to the scanning area $A_{\text {scann }}$, dependent on the height $h$ of the roughness profile, and is therefore also dependent on scale. Figure $\mathbf{8}$ exemplifies a bearing analysis for the flattened AFM image of the spherical cap of a tin particle. 
locations, an upper and lower limit of the theoretical adhesion force was calculated. The lower limit was computed using the $90 \%$ percentile of particle and substrate rms roughness in Eq. (8), whereas for the upper limit, the $10 \%$ percentile (upper and lower error bar in Fig. 7) was used. Based on estimations of the circular contact area between particle and substrate, with Eq. (11) we assume the box plot at $75 \times 75 \mathrm{~nm}$ scanning area in Fig. 7 to be the best representation of the contact area. The effect of micro-asperity plastic flattening at the particle, caused by alternating contact forces of the vibration method, was considered through the modified effective roughness $\mathrm{rms}_{12 \text { _plast }}$ of Eq. (9), taking into account a reduced area of contact stemming from surface roughness as described in Section 4.1. For theoretical predictions of the maximum contact pressure force $\mathrm{F}_{\text {contact_max }}$ of Eq. (13), we used twice the van der Waals adhesion force $\mathrm{F}_{\mathrm{vdW}}$ of the unstressed system of Eq. (6) as described in Section 2.1.2, assuming particle detachment for $\left|\mathrm{F}_{\mathrm{vdW}}\right| \leq$ $\left|\mathrm{F}_{\text {detach }},\right| \mathrm{F}_{\text {detach }}|\approx| \mathrm{F}_{\text {contact }} \mid$ and $\left|\mathrm{F}_{\text {contact_max }}\right| \approx\left|\mathrm{F}_{\text {vdW }}\right|+\left|\mathrm{F}_{\text {contact }}\right|$. The surface-energy-based approaches of DMT as well as JKR overestimate the measured values.

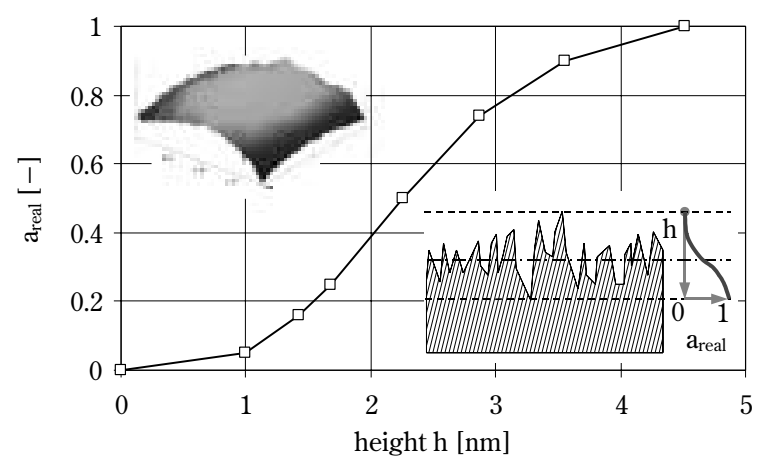

Fig. 8 Bearing analysis for a tin particle based on a flattened AFM image of $250 \times 250 \mathrm{~nm}$ area and schematic of the bearing procedure (lower right picture).

\section{Experimental Results and Discussion}

The results of the vibration method represent the pull-off forces between various particles, each at different substrate locations. In contrast, the results of the colloidal probe technique (referred to as AFM) represent either repeated measurements between one particle at the same substrate location (referred to as 1 location) or at 200 different locations of the substrate (matrix). For a theoretical prediction based on van der Waals adhesion, we used the Rabinovich model of Eq. (6). To account for the variations in surface roughness of different particles and substrate

\subsection{Tin particles on electropolished stainless steel substrates}

Pull-off forces for tin spheres on an electropolished stainless steel substrate are shown in Figure 9. The colloidal probe technique (AFM) (Lehrstuhl für Maschinen und Apparate Kunde (MAK), TV München) and vibration method show relatively good agreement. For small particle sizes, the results of both methods are for the most part within the range defined by the

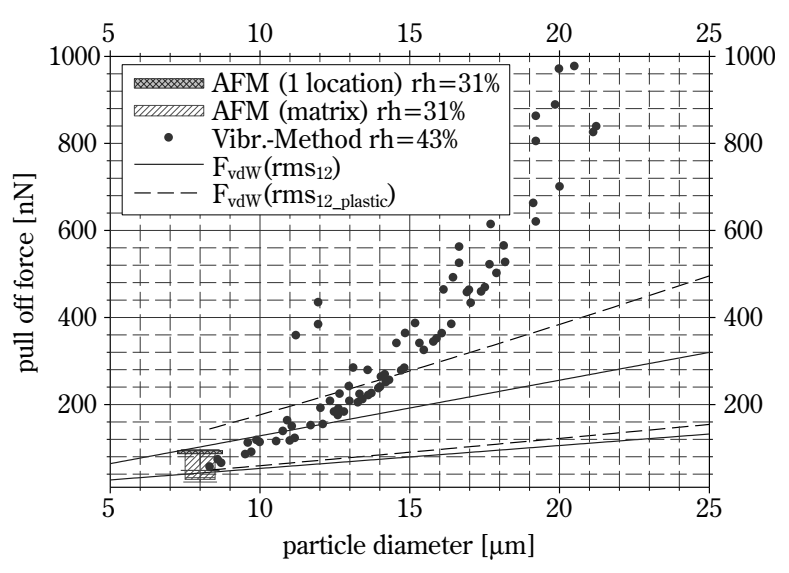

Fig. 9 Pull-off force for tin spheres on electropolished stainless steel substrates and predicted upper and lower limit of van der Waals adhesion using Eq. (6) with $\mathrm{rms}_{12 \text { upper }}=0,54 \mathrm{~nm}$ and $\mathrm{rms}_{12 \_ \text {lower }}=1,05 \mathrm{~nm}$ of Eq. (8) and $\mathrm{rms}_{12 \_ \text {plastic }}$ of Eq. (9). 
Rabinovich approach of Eq. (6), using the 90\% and $10 \%$ percentile of particle and substrate rms roughWness in Eq. (8) as a lower and upper limit of the predicted van der Waals adhesion force. With increasing particle size, the adhesion forces measured with the vibration method exhibit a stronger increase than predicted, showing a tendency to lower rms roughness values of the harder substrate with the higher Young's Modulus and hardness. Since the vibration of the substrate also causes contact forces between particle and substrate of the same order, higher adhesion forces and particle diameters represent higher contact forces. It is therefore possible that micro-asperities of the tin spheres with the comparatively lower Young's Modulus and hardness undergo an increasing flattening with increasing particle diameter, whereas the harder asperities of the substrate remain unchanged. This would result in a lower rms value which corresponds to a more intimate contact and an increase in adhesion force. To consider possible micro-asperity plastic flattening, the modified rms value $\mathrm{rms}_{12}$ plastic of Eq. (9) was used in Eq. (6). Especially for higher particle diameters and contact forces, the adhesion force intensification observed with the vibration method cannot be explained by plastic flattening only. Further reasons for this effect might be seen in an increase in the points of contact caused by rearrangements and elastic flattening of the particle not incorporated in the approach. Furthermore, the influence of the high-frequency alternating stresses might prevent a relaxation of the elastically flattened asperity, causing a kind of visco-elastic behaviour. Pull-off force measurements with the colloidal probe technique at an elevated measuring frequency of $5 \mathrm{~Hz}$ presented by Zhou [25] showed a significant adhesion force intensification with increasing measuring sequence. Resonant particle oscillation before particle detachment might be another source of adhesion force intensification. It can be caused by sinusoidally alternating elastic deformation of micro-asperities through high-frequency alternating detachment and contact forces of the vibration method for $F_{\text {adhesion }}>F_{\text {detach }}$. Estimations of this effect based on approaches by Ziskind [26] will be presented elsewhere.

Figure 10 shows successive pull-off force measurements for two tin particles on an electropolished stainless steel substrate under variation of the contact force measured with the colloidal probe technique (MPI for Polymer Research, Mainz, Germany) at $1 \mathrm{~Hz}$ measuring frequency. The measuring sequences tend toward increasing adhesion force with increasing contact force. Variations in the surface roughness of

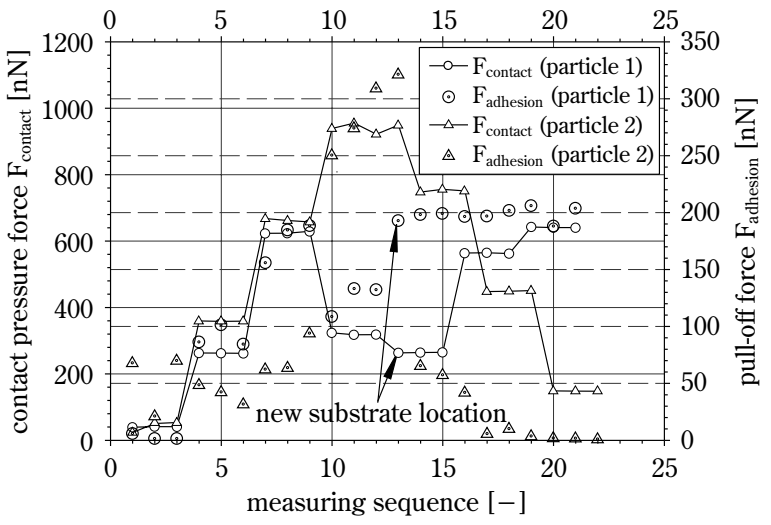

Fig. 10 Pull-off force for two tin particles on electropolished stainless steel substrates under variation of the contact force measured with the colloidal probe technique (particle 2 measured at 2 different substrate locations; particle 4 measured at the same location).

particle and substrate dependent on the substrate location influence the intensity of this effect and cause variations in the measured pull-off force for equal contact forces. Variations might also be attributed to impurities remaining on the adhesion partners after the cleaning procedure described in Section 3. In order to match the practical use of the surfaces, no further cleaning procedures were applied. After reducing the applied contact force, the measured pull-off force also decreased, indicating no significant plastic deformation of the micro-asperities.

\subsection{Tin particles on ground stainless steel substrates}

Figure 11 presents the pull-off forces for tin spheres on a ground stainless steel substrate. Again, the results of the vibration method and the colloidal probe technique (MAK, TV München) show relatively good agreement. In contrast to the comparatively smooth electropolished substrate, both measuring techniques show stronger variations. These fluctuations are primarily caused by large variations of the surface roughness of the ground substrate dependent on the location (Fig. 7). Compared to the electropolished substrate, adhesion force intensification caused by contact forces of the vibration method as explained in Section 5.1 can be observed on a smaller scale, which might be attributed to the higher surface roughness of the substrate. Since adhesion force intensification is not considered in the approach represented by Eq. (6) which relies on the rms roughness of particle and substrate $\mathrm{rms}_{12}$ only, the measured pull-off forces 


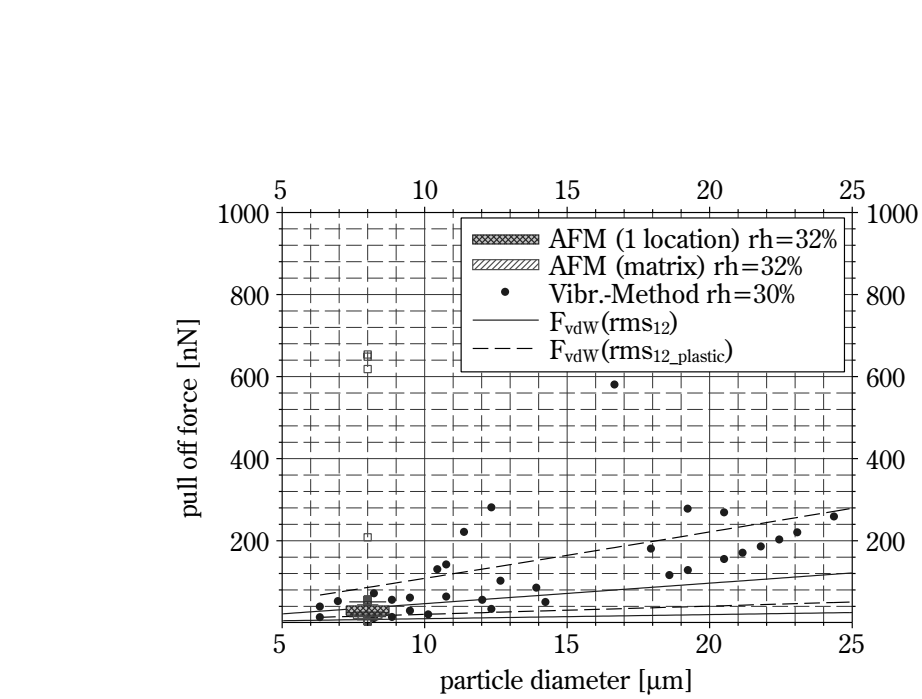

Fig. 11 Pull-off force for tin spheres on ground stainless steel substrates and predicted upper and lower limit of van der Waals adhesion using Eq. (6) with $\mathrm{rms}_{12 \text { upper }}=0,7 \mathrm{~nm}$ and $\mathrm{rms}_{12 \_ \text {lower }}=1,9 \mathrm{~nm}$ of Eq. (8) and $\mathrm{rms}_{12 \_ \text {plastic }}$ of Eq. (9).

are underestimated. Another reason might be seen in the existence of a microscopic roughness scale on the ground substrate superimposed on the macroscopic roughness scale as described in Section 3.1, since the "non-contact" force between the macroscopic asperity and the surface is neglected. The use of an approach which accounts for this additional interaction [9] might improve the approximation. The approximation of the results with Eq. (6) can be improved considering possible micro-asperity plastic flattening with the modified rms value rms $_{12 \text { _plastic }}$ of Eq. (9).

Successive pull-off force measurements for two tin particles on a ground stainless steel substrate under variation of the contact force measured with the colloidal probe technique (MPI for Polymer Research, Mainz, Germany) are presented in Figure 12.

Compared with the measuring sequences on the electropolished substrate (Fig. 10), adhesion force intensification caused by increasing contact forces can be observed on a much smaller scale. This tendency is in agreement with our measuring results with the vibration method. Again, variations in particle and substrate surface roughness dependent on the substrate location have a great influence on the intensity of this effect.

\subsection{Dependency off the pull-off force on the contact time}

As already mentioned in Section 2.2, the differences in the contact time between particle and substrate before particle detachment might lead to diverging measuring results for the same particle-substrate system caused by visco-elastic effects [10, 21]. For that

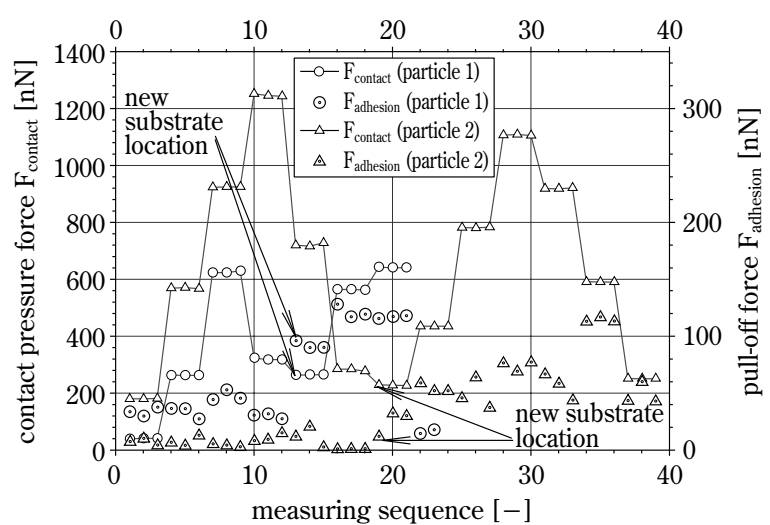

Fig. 12 Pull-off force for two tin particles on ground stainless steel substrates under variation of the contact force measured with the colloidal probe technique.

reason, the pull-off force was measured with the colloidal probe technique (MPI for Polymer Research, Mainz) at contact times of $1 \mathrm{~s} \mathrm{(1} \mathrm{Hz}$ measuring frequency) and $30 \mathrm{~s}$ under a given contact pressure force. Figure $\mathbf{1 3}$ shows the results of pull-off force measurements for tin particles on ground and electropolished stainless steel substrates at various substrate locations under variation of contact time and contact force. Within this time scale, no significant adhesion force intensification caused by increasing contact time at several contact forces was observed.

\subsection{Glass particles on silicon wafer substrate}

The pull-off force for glass particles on a silicon

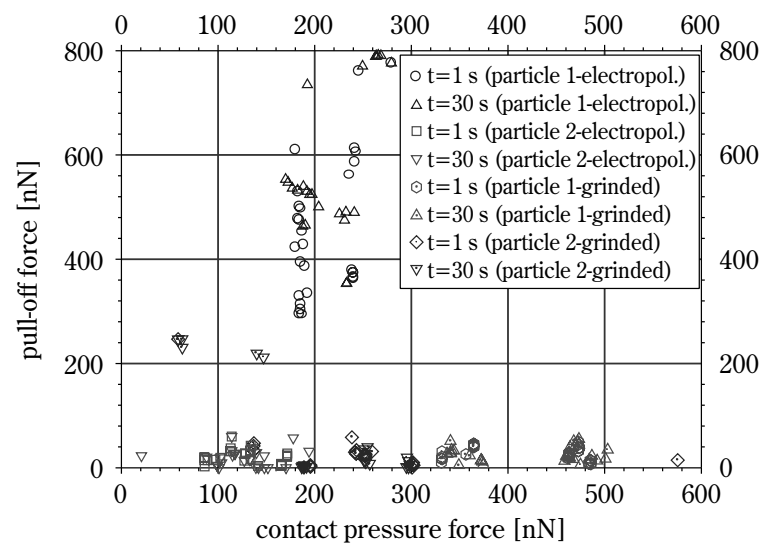

Fig. 13 Pull-off force for two tin particles on ground and electropolished stainless steel substrates at various substrate locations under variation of contact time and contact force measured with the colloidal probe technique. 
vedef rolbstrate measured by the vibration method and the colloidal probe technique (MAK, TV München)

Ware shown in Figure 14. For small particle sizes, both methods show relatively good agreement. With increasing particle size, the vibration method exercises higher pull-off forces than the colloidal probe technique. Possible explanations for this effect were already described in Section 5.1. Analogous to the results for tin particles on the electropolished substrate, the comparatively smooth substrate seems to increase the effect of adhesion force intensification. The higher hardness and Young's Modulus (Table 1) of the glass particles in comparison with the tin particles reduce this effect. For small particle sizes, the results of the vibration method are generally within the range of the Rabinovich approach of Eq. (6). With increasing particle diameter and contact forces, the pull-off forces measured with the vibration method exceed the theoretical values for the unstressed system. With the modified rms roughness of Eq. (9), the adhesion force intensification observed with the vibration method can be explained - to some extent - by plastic flattening.

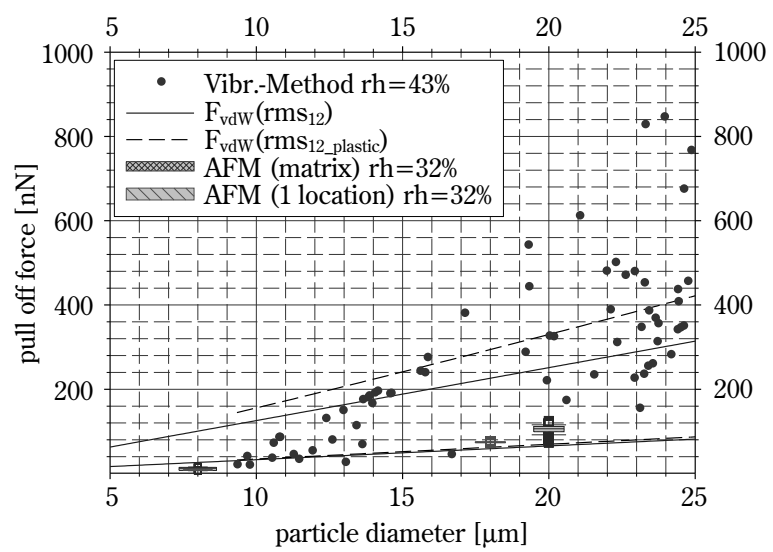

Fig. 14 Pull-off force for glass spheres on silicon wafer substrates and predicted upper and lower limit of van der Waals adhesion using Eq. (6) with $\mathrm{rms}_{12 \_ \text {upper }}=0,38 \mathrm{~nm}$ and $\mathrm{rms}_{12 \_ \text {lower }}=0,97 \mathrm{~nm}$ of Eq. (8) and $\mathrm{rms}_{12 \_ \text {plastic }}$ of Eq. (9).

\section{Conclusions}

Pull-off forces were measured using the vibration method and the colloidal probe technique. Both methods show relatively good agreement for small particle sizes. Alternating contact forces of the vibration method can cause an increase in the adhesion force through flattening of micro-asperities. This effect was observed on a larger scale for the substrates with a comparatively smaller rms roughness and also found to be dependent on the mechanical properties of particle and substrate. Pull-off force measurements with the colloidal probe technique on the same adhesion systems with special attention to the influence of contact force also tended toward increasing adhesion force with increasing contact forces, exposing no significant indication of micro-asperity plastic flattening. Conforming with the results of the vibration method, the adhesion force intensification caused by increasing contact force was observed on a much smaller scale for the ground metal substrate with higher rms roughness values. At several contact forces, no significant adhesion force intensification caused by increasing contact time of $30 \mathrm{~s}$ was observed. Variations of the measuring results which were observed with both measuring techniques are predominantly caused by variations of the surface roughness, which in turn depend on the particle and substrate location. For small particle sizes, the results of both methods are generally within the range defined by the Rabinovich approach, using the $90 \%$ and $10 \%$ percentile of particle and substrate rms roughness as a lower and upper limit of the predicted van der Waals adhesion force. With increasing particle size, the adhesion forces measured with the vibration method show a stronger increase than predicted. The possible effect of microasperity plastic flattening of the particle was considered through the modified effective roughness in the Rabinovich approach, resulting in a better approximation of the results. Especially for higher particle diameters and contact forces, the adhesion force intensification observed with the vibration method cannot be explained by plastic flattening only. Further reasons for this effect might be seen in an increase of the points of contact caused by rearrangements or high-frequency alternating stresses which might prevent a relaxation of the elastically flattened asperity, thus causing visco-elastic behaviour.

\section{Acknowledgements}

We express our gratitude to the Deutsche Forschungsgemeinschaft, which supported this work within the Research Programme Ri 776/9-3. We are furthermore grateful to Dr. Michael Kappl and Dr. Lars Oliver Heim (MPI for Polymer Research, Mainz, Germany) and Dr. Bernhard Weigl (Lehrstuhl für Maschinen- und Apparatekunde, TU München, Germany) for performing force measurements with the 
prial probe technique.

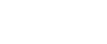

\section{Nomenclature}

a : acceleration

A : Hamaker constant

AFM : atomic force microscope

$\left[\mathrm{m} / \mathrm{s}^{2}\right]$

$\mathrm{d}_{\mathrm{P}} \quad$ : particle diameter $\quad[\mu \mathrm{m}]$

E :Young's Modulus [GPa]

$\mathrm{f}$ : frequency $[\mathrm{Hz}]$

$\mathrm{F}_{\mathrm{ad}} \quad$ : adhesion force

$\mathrm{F}_{\text {contact }}$ : inertia force $=$ contact force for $\pi<\omega \mathrm{t}<2 \pi$

$F_{\text {detach }}$ : inertia force $=$ detachment force for $0<\omega \mathrm{t}<\pi$

$\mathrm{F}_{\text {grav }}$ : gravitational force

$F_{\text {inertia }}$ : inertia force

$\mathrm{H}$ : hardness

$\mathrm{h} \quad$ : flattening

$\mathrm{K}_{\text {elast }} \quad: \mathrm{K}_{\text {elast }}=\frac{4}{3} \cdot\left(\frac{1-v_{\mathrm{P}}^{2}}{\mathrm{E}_{\mathrm{P}}}+\frac{1-v_{\text {sub }}^{2}}{\mathrm{E}_{\text {sub }}}\right)^{-1}$

$[\mathrm{Pa}]$

LSV : laser scanning vibrometer [-]

$\mathrm{m}_{\mathrm{P}} \quad$ : particle mass [kg]

$\mathrm{Re}_{\text {channel }}$ : Reynolds number of the flow channel [-]

rms : root mean square [nm]

SEM : scanning electron microscope [-]

$\mathrm{T}$ : temperature $\quad\left[{ }^{\circ} \mathrm{C}\right]$

$\mathrm{t}$ : time [s]

$\mathrm{U}_{\mathrm{ex}} \quad$ : excitation voltage of the actuator [V]

$v \quad$ : Poisson's ratio $\quad[-]$

$\rho \quad$ : density $\quad\left[\mathrm{gcm}^{-3}\right]$

$\omega \quad$ : angular frequency $\quad[\mathrm{Hz}]$

\section{References}

1) W.A. Ducker, T.J. Senden, R.M. Pashley: Direct measurement of colloidal forces using an atomic force microscope, Nature 353 (1991), 239-241.

2) H.-J. Butt: Measuring electrostatic, van der Waals, and hydration forces in electrolyte solutions with an atomic force microscope, Biophys. J. 60 (1991), 1438-1444.

3) G. Binning, C.F. Quate, C. Gerber: Atomic force microscope, Phys. Rev. Lett. 56 (1986), 930-933.

4) R.I. Larsen: The adhesion and removal of particles attached to air filter surfaces, Am. Indust. Hyg. J. 19 (1958), 265-270.

5) H. Krupp: Particle adhesion. Theory and experiment, Adv. Coll. Interf. Sci., 1 (1967), 111-239.

6) B.V. Deryaguin, A.D. Zimon: Adhesion of Powder Particles to Plane surfaces, Kolloidnyi Zhurnal, Vol. 23, No. 5
(1961), 544-552.

7) K. Hein, T. Hucke, M. Stintz, S. Ripperger: Analysis of Adhesion Forces between Particles and Wall based on the Vibration Method, Part. Part. Syst. Charact. 19 (2002), 269-276.

8) D. Leigthon, A. Avcrivos: The lift on a small sphere touching a plane in the presence of a simple shear flow, J. Appl. Phys. (ZAMP) 36 (1985), 174-178.

9) M.E. O'Neill: A sphere in contact with a plane wall in a slow linear shear flow, Chem. Eng. Sci. 23 (1968), 12931298.

10) H. Rumpf, K. Sommer, K. Steier: Mechanismen der Haftkraftverstärkung bei der Partikelhaftung durch plastisches Verformen, Sintern und viskoelastisches Fließen, Chem.-Ing.-Techn., 48 (1976), 300-307.

11) M. Kappl, H. Butt: The Colloidal Probe Technique and its Application to Adhesion Force Measurements, Part. Part. Syst. Charact. 19 (2002), 129-143.

12) Y.I. Rabinovich, M. Moudgil et al.: Adhesion between nanoscale rough surfaces, J. Colloid Interface Sci., 232 (2000), 10-24 .

13) H. Rumpf: Die Wissenschaft des Agglomerierens. Chem.-Ing.-Tech. 46 (1974), 1-11.

14) J.D. Kiely, D.A. Bonnell: Quantification of topographic structure by scanning probe microscopy, J. Vac. Sci. Technol. B15 (4), 1483-1493 (1997).

15) H.C. Hamaker: Physica 4 (1937), 1058.

16) B.V. Deryaguin, V.M. Muller, Y.P. Toporov: Effect of Contact Deformations on the Adhesion of Particles, J. Coll. Int. Sci. 53 (2), 314 (1975).

17) K.L. Johnson, K. Kendall, A.D. Roberts: Surface energy and the contact of elastic solids, Proc. R. Soc. London A 324, 301 (1971).

18) Y.I. Rabinovich, J.J. Adler, M.S. Esayanur, A. Ata, R.K. Singh, B.M. Moudgil: Capillary forces between surfaces with nanoscale roughness, Adv. Coll. Int. Sci. 96 (2002), 213-230.

19) K. Cooper et. al: Simulation of Adhesion of Particles to Surfaces, J. Coll. Int. Sci. 234 (2001), 284-292.

20) W. Peukert, C. Mehler, M. Götzinger: Application of Adsorption and Adhesion Measurements for Particle Surface Characterization, Part. Part. Syst. Charact. 18 (2001), 229-234.

21) Wei Hsuin Yang: The contact problem for viscoelastic bodies, J. Appl. Mech., Trans. ASME 33 (1966), 395-401.

22) L. Kogut, I. Etsion: Adhesion in elastic-plastic spherical microcontact, J. Coll. Int. Sci. 261 (2003), 372-378.

23) D. Maugis, H.M. Pollock: Surface Force, Deformation and Adherence at metal microcontacts, Acta metal. 32 (1984), 1323-1334.

24) H.J. Hertz: Reine Angewandte Math. 92, 156 (1881).

25) H. Zhou et. al: Tailoring Particle-Substrate Adhesion, Oral Presentation 25.5 at the International Congress for Particle Technology 2004, Nuremberg.

26) G. Ziskind: Particle Behaviour on Surfaces subjected to external excitations, J. Aerosol Sci., Vol. 31, No. 6 (2000), 703-719. 
an $a^{r}$

\section{Author's short biography}

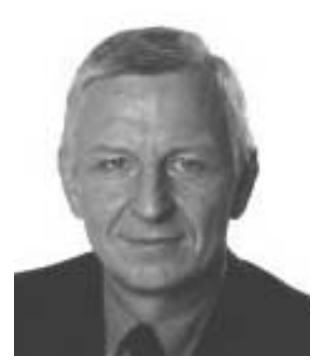

\section{Siegfried Ripperger}

Born 1950 in Bous/Saarland. Studied mechanical and chemical engineering at the University of Applied Science at Saarbrücken and at the University of Kaiserslautern. Dr.-Ing. 1982 at Kaiserslautern with a thesis on the adsorption of organic compounds and water out of air at activated charcoal. 1983-1989 with Akzo AG at Wuppertal. Since 1987 head of the development and application department for technical membranes.

1989-1993 professor for chemical engineering and environmental technology at the University of Applied Science at Fulda. Since 1990 dean of the Faculty of Food Technology. 1993-2004 professor at the Technical University of Dresden, chair for mechanical process engineering. Since 1997 director of the Institute of Chemical Engineering and Environmental Technology at Dresden. Since April 2004 professor at the Technical University of Kaiserslautern, chair for mechanical process engineering.

More than 140 publications. Main research activities: the separation of fine particles out of gases and liquids, membrane separation, in-line particle measurement, characterization of dispersed systems and product development.

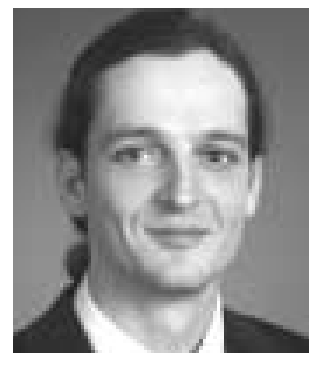

\section{Konrad Hein}

$\mathrm{K}$. Hein graduated in process engineering from the Technical University of Dresden, Germany, in 1999. After carrying out his diploma thesis on the detection of small amounts of coarse particles in highly concentrated suspensions using a laser backscattering technique, he started working as a research assistant on a Ph.D. project in Prof. Ripperger's process engineering group at the Technical University of Dresden. His project deals with particle adhesion phenomena on vibrating surfaces in air. 